\title{
Asentamiento y paisaje. Un estudio de caso mediante el análisis de cuencas visuales teóricas en el Valle de Balcosna, provincia de Catamarca, Argentina
}

\section{RMA}

Arqueología
Settlement and landscape. A case study through the analysis of visual
theory basins in the valley of Balcosna, province of Catamarca, Argentina

Emilio Alejandro Villafañez

Escuela de Arqueología, Universidad Nacional de Catamarca. Maximio Victoria 55, predio universitario (K4700), San Fernando del Valle de Catamarca. E-mail: emilio81@gmail.com

\begin{abstract}
Resumen
Este trabajo tiene como finalidad acercar al lector un estudio de caso sobre cuencas visuales teóricas en el Valle de Balcosna, provincia de Catamarca. Este lugar, ubicado en las estribaciones finales de las yungas, cuenta con muy pocas referencias en la bibliografía arqueológica, por lo que nuestras primeras preguntas fueron guiadas por la necesidad de conocer cómo fue la construcción social del paisaje en el valle, donde la visibilidad parece haber sido un factor importante en la sociabilidad de los grupos que habitaron la zona. Metodológicamente llevamos a cabo prospecciones guiadas por las sendas del valle, lo cual nos permitió conocerlo desde una perspectiva novedosa. Interpretamos a este valle como un lugar donde el paisaje fue constantemente re-creado, con sitios dispuestos siguiendo una lógica donde la visibilidad e inter-visibilidad fueron factores relevantes y hasta necesarios en la constante interacción social de las personas que habitaron la zona.
\end{abstract}

Palabras clave: Visibilidad; Sendas; Paisaje; Valle de Balcosna.

\begin{abstract}
This paper aims to bring the reader a theoretical case study of the viewshed in the Balcosna Valley (Catamarca province). This place is located in the last slopes of the Yungas zone, and it counts with few references in the archaeological literature, so our first questions were directed by the need to understand the social construction of landscape in the valley, where visibility seems to have been an important sociability of groups that inhabited the area. Methodologically we conducted archaeological surveys guided by the trails which still persist in the valley, which allowed us to comprehend them from a novel perspective. We interpret this valley as a place where the landscape was constantly re-created, where the sites were arranged in a logic where visibility and inter-visibility were relevant factors and also to a point necessary in the constant social interactions of the persons inhabiting the area.
\end{abstract}

Keywords: Visibility; Paths; Landscape; Balcosna valley.

El Valle de Balcosna se encuentra en el extremo septentrional del Departamento Paclín, Provincia de Catamarca, Argentina. Las áreas cercanas más reconocidas por la arqueología regional sin duda son el Valle de Ambato al Oeste, el Campo del Pucará al Norte y los llanos de Alberdi al Este (ver figura 1). Aunque estos han sido ampliamente estudiados desde hace varias décadas, no ha sucedido lo mismo con Balcosna.

En relación a sus características ambientales, pese a que es un área pequeña en términos geográficos y regionales, se destaca por ser muy variada. Se ubica en la Provincia Fito-geográfica de las Yungas (ver Cabrera 1976) y posee tres distritos principales: 1) las Selvas Montanas, sobre el extremo Norte; 2) los Bosques Montanos, que abarcan la zona baja de todo el valle hasta los 1600 msnm; y 3) los Pastizales de Altura, que llegan desde dicha altura hasta punta de cumbre a los 1950 msnm. La parte más boscosa y exuberante, definida como Selvas Montanas tiene límites muy marcados y definidos, llegando a cubrir una parte muy exigua de nuestra área de estudio. Por otra parte los Bosques Montanos, en la actualidad, han sido muy alterados por el régimen de cultivos y ganadería extensiva de la zona, lo que ha producido que solo queden elementos propios de dicho distrito en lugares acotados y puntuales, como quebradas y zonas elevadas. Por último, los Pastizales de Altura (de yungas) no han sufrido ninguna clase de alteración evidente, aunque debe destacarse que en zonas muy altas nos encontramos con la hibridación propia del choque de esta Provincia Fito-geográfica con la Chaqueña, lo que produce una suerte de mezcla entre ambas (Morlans 1995; Cattania y Varela 2010). Podemos 


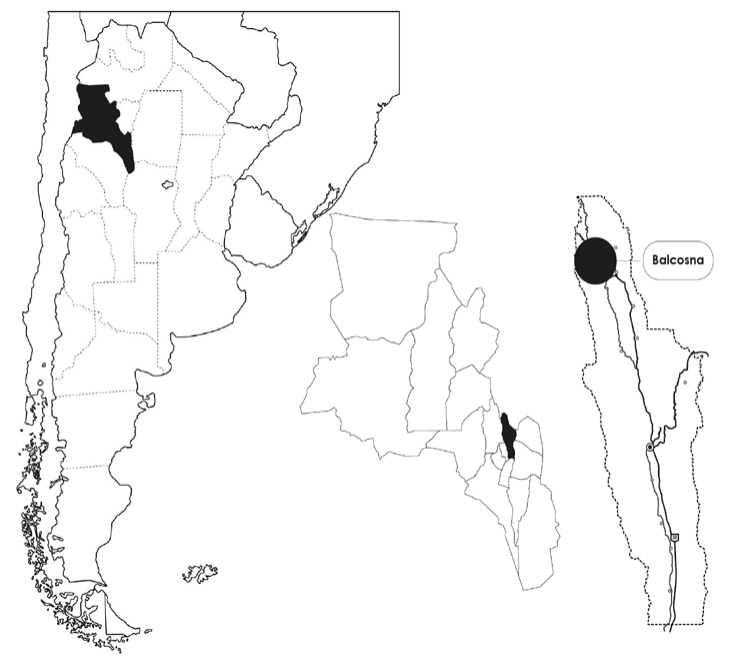

Figura 1: Ubicación del área de estudio respecto a la provincia de Catamarca y la República Argentina.

Figure 1: Location of the study area in the province of Catamarca and the Argentine Republic.

decir que el Valle de Balcosna forma parte de uno de los ramales finales de las yungas, es decir, aunque no esté completamente en ellas se encuentra en sus puertas, como ocurre también en relación a su vinculación con parte del ambiente típico del Chaco Serrano.

La arqueología del Valle de Balcosna comenzó a conocerse recién en los últimos años, siendo tratada de manera parcial (González 1977; Barrionuevo y Schobinger 1992; Kriscautzky 1995; 2006), con la descripción de hallazgos puntuales o menciones casi especulativas acerca de su arqueología. Nunca se habían Ilevado a cabo en él investigaciones concretas, por lo que este estrecho valle perteneciente a la zona de Yungas por mucho tiempo ha sido un interrogante para la arqueología regional. Esto se debe principalmente a la escasez de investigaciones sobre la vertiente oriental en la que se incluye Balcosna (Albeck 1994; Garay de Fumagalli 1994; Ventura 1994, 2001; entre otros).

Teniendo en claro lo antes mencionado, debemos comenzar señalando que nuestras investigaciones se iniciaron en el año 2005 y dos años después se condensaron y sistematizaron en una Tesis de Licenciatura (Villafañez 2007). En aquella oportunidad, seguimos los postulados de la Arqueología Espacial (Clarke 1977; Hodder y Orton 1990), realizando un muestreo no probabilístico del área para elegir cuatro zonas topográfica y ambientalmente distintas. Esta estrategia posibilitó encontrar 41 sitios arqueológicos, junto con varios sistemas agrícolas y de manejo y control del agua. En nuestras primeras interpretaciones, expusimos que existía un patrón de asentamiento de estructuras dispersas de tamaño y forma variable, en las cuales se habrían llevado a cabo diversas actividades, que no mostraban ningún grado de subordinación respecto a las áreas vecinas, aunque sí posibles vínculos regionales (Villafañez 2007).

En esta publicación exponemos los nuevos trabajos de prospección, lo que nos ha permitido ampliar nuestro conocimiento sobre cómo se conformó el paisaje arqueológico del valle para momentos de la "Cultura de La Aguada" (sensu González 1961-4). En cuanto a la cuestión temporal, si bien existen pocos datos concretos, se trataría de un grupo humano que se asentó en el valle a mediados del primer milenio ${ }^{1}$ de la era, atentos a la datación de carbono 14 que hemos realizado en el sitio 111 , un recinto cuadrangular con dos recintos y un patio.

En esta ocasión nos sumamos a la hipótesis de que la visibilidad es un factor relevante para los grupos humanos, llegando a ser un elemento constituyente de la sociabilidad (Llobera 2003). Por ello realizamos el análisis de cuencas visuales teóricas para evaluar si los modos de emplazamiento del valle siguieron alguna lógica específica en relación a los grados de visibilidad e inter-visibilidad entre los sitios y el paisaje.

\section{Sobre el paisaje y la visibilidad}

En trabajos previos (ver Villafañez 2010, 2012, 2013; Villafañez et al. 2015), nos centramos en una propuesta teórica que parte desde la Geografía Humana y la Arqueología del Paisaje, algo que pretendemos seguir haciendo, dado el hecho de que para nosotros la transdisciplinariedad entre ambas ciencias nos permite tener un acercamiento tanto teórico (en relación a la noción de paisaje) como también metodológico (en relación a los usos del SIG, como se verá en este trabajo).

Desde los años '70 el Ilamado giro espacial generó un acercamiento de la primera a otras ciencias sociales, haciendo de la transdisciplinariedad y la interdisciplinariedad una pieza clave para que Hiernaux y Lindón (2006) lo entiendan como una espacialidad explosiva. Esto llevó a considerar a la materialidad espacial como un aspecto significante en las relaciones sociales (Sanguin 1981; Nogué i Font 1985), lo cual consistió en un paso fundamental para la renovación crítica de la Geografía Humana y la Arqueología Post-Procesual, sirviendo de punto de partida para entender al paisaje como un nuevo objeto de estudio social (Villafañez 2011). Sin lugar a dudas, la interdisciplinariedad entre la Geografía Humana y la Arqueología del Paisaje nos brinda importantes herramientas teórico-metodológicas como punto de partida.

No es de nuestro interés hacer un recorrido histórico sobre las nociones de espacio y paisaje en arqueología, algo que está por demás explicitado en casi todas las

'Las muestras han proporcionado una ubicación temporal del recinto en el siglo VII DC, cuya datación por AMS arrojó una edad de 644 $+/-80$ d.C $(p=0,95)$ con un sigma Laboratorio de "NSF Arizona AMS Facility. The University of Arizona EE.UU". 
publicaciones que realizan trabajos de este estilo, aunque si queremos resaltar qué entendemos por paisaje, para así concentrarnos de manera más concisa en las nociones sobre visibilidad.

Hablar de paisaje es hacer referencia a un espacio socialmente producido, habitado y significado y no a un simple contenedor de la acción. No se trata de un mero espacio geométrico donde el principal problema es la distancia y la fricción, sino que es un espacio productor de la vida social. Además el paisaje se aborda metodológicamente desde la escala humana, teniéndose en cuenta las prácticas que las personas desarrollan, las relaciones sociales que entablan y las experiencias que viven en estos espacios significados (Acuto 2013).

Adentrándonos sobre la visibilidad, un paisaje visual puede ser definido como la articulación de las propiedades visuales generadas por (o asociada a) una configuración espacial específica (Llobera 2003). En las últimas décadas se ha empezado a estudiar la visibilidad como un factor relevante para los grupos humanos, en cuanto que diferentes formas de organización humana permiten registrar diversas pautas visuales, las cuales evolucionan al mismo tiempo que las sociedades cambian, volviéndose en muchos casos un elemento de prestigio social (Fábrega Álvarez 2005; García Sanjuán 2006).

Criado Boado (1995) parte de la premisa de que, en parte, las producciones materiales de las sociedades están realizadas para ser vistas y tienen una estrategia de visibilización. Esta intención está estrechamente vinculada con el tipo de racionalidad espacial vigente en un determinado contexto cultural, "...pues, si tenemos en cuenta que la forma de visibilizar los productos humanos altera el paisaje, la opción por una u otra estrategia de visibilización o invisibilización propone una determinada actitud hacia el entorno" (Criado Boado 1995:100).

Lo cierto es que la visibilidad es un elemento de gran importancia a tener en cuenta. El hecho de poder ver y ser visto, para las sociedades pasadas, podría haber implicado tener un mayor control y seguridad dentro de un asentamiento, reforzándola internamente; podría haber servido para fortalecerla ante amenazas de agresiones; o para controlar algunos recursos básicos, lugares significativos como campos de cultivo, canteras, vías de circulación, etc. Una mejor visibilidad, asimismo, podría haber significado poder realzar las propiedades de algún monumento, dándole una dimensión escénica más relevante ante otras estructuras (Criado Boado 1993; García Sanjuán 2006).

En cuanto a los estudios realizados con SIG, estos se han convertido en una herramienta ampliamente utilizada en el mundo de la arqueología², además ha tomado

${ }^{2}$ Existe una amplia bibliografía respecto a la relación de los SIG y el análisis espacial, Conolly y Lake en la introducción de su conocido libro se preguntan sobre el debate en cuanto a si los SIG son una metodología o una ciencia en sí misma, ellos nos dicen que: "el tema fuerza en la última década en trabajos de habla hispana (Lanzelotti 2015; Figuerero Torres e Izeta 2011; Wynveldt et al 2013; Parcero Oubiña et al. 2009; Grau Mira 2006, 2011; entre otros).

Los análisis de visibilidad son llevados a cabo por una cuenca visual teórica, entendida ésta como "el conjunto de todas las localizaciones o puntos del territorio que son visibles desde un punto de observación específico, dada una distancia máxima de visión, y en base únicamente a la topografía" (García Sanjuán 2006: 184). Este procedimiento se llevó adelante en nuestra zona de estudio mediante el empleo de un modelo digital de elevación (MDE) raster de $30 \mathrm{~m}$ el pixel (tomado desde la página web de la NASA), de forma que el cálculo permitió definir qué celdas son visibles desde el punto de observación establecido y cuál es el perímetro de visión máximo. Una vez establecido el punto desde dónde se lleva adelante el análisis, el algoritmo calcula un nuevo raster binario, donde las celdas con valor 1 son visibles y las de valor 0 son invisibles (García Sanjuán 2006).

Existen diversos factores que intervienen en los cálculos de cuenca visual en SIG. Algunos de ellos pueden (y en algunos casos deben) ser debidamente manipulados para obtener resultados que se asemejen mejor a la hora de reconstruir los espacios sociales que estamos estudiando. Para nuestro caso prestaremos especial atención a:

a) La altitud sobre la que está situado un determinado punto y su relación con el entorno que le rodea. Este factor se obtiene del modelado digital del terreno sobre el que se sitúa el punto, en este caso cada yacimiento.

b) El azimut, ángulo horizontal que limita la exploración, que en este trabajo se ha establecido en $360^{\circ}$.

c) El ángulo vertical que limita la exploración. El plano horizontal, 0 grados, se sitúa en la cota del punto (yacimiento). En este caso se han establecido los ángulos superior e inferior en $90^{\circ}$ y $-90^{\circ}$ respectivamente.

d) Los radios mínimos y máximos que limitan la búsqueda de áreas visibles. En los estudios de visibilidad teórica suele considerarse que el límite del umbral de la visión humana fiable, es decir, con un nivel de detalle aceptable, se sitúa rondando los tres kilómetros de distancia (la cual supone una cuenca visual teórica máxima de 28,2km) (García Sanjuán 2006), criterio que hemos seguido a la hora de delimitar el campo de visión.

es importante porque si es SIG es sólo una herramienta, entonces su utilización puede considerarse básicamente neutral desde el punto de vista de la teoría, pero si es una ciencia su utilización conlleva automáticamente una determinada perspectiva teórica, que puede gustar o no" (Conolly y Lake 2009: 17,18). Aunque no nos interesa profundizar aquí estas cuestiones sí coincidimos con estos autores en cuanto a que "la clave del éxito consiste en utilizar el SIG de una forma adecuada, lo que significa no perder de vista sus lastres teóricos y tener un adecuado dominio técnico de las muchas y poderosas posibilidades que ofrece (Conally y Lake 2009:27). 
e) La altura del punto de observación. Ésta se encuentra referida a la altura sobre el terreno a la que se encuentra el punto de observación. En este estudio la hemos situado a 1,65 metros de altura, ya que es la altura definida desde la que una persona puede alcanzar sin demasiado esfuerzo a observar el paisaje salvando los obstáculos visuales más próximos y de menor entidad.

En relación a nuestra investigación, debemos decir que la técnica de prospección utilizada y el conocimiento de campo que hemos adquirido en el transcurso de estos años, sumado a la detallada descripción de los asentamientos y el paisaje circundante, ha hecho posible que podamos adentrarnos en las pautas de visibilidad desde dos lugares ${ }^{3}$ : en una primera instancia, gracias a los análisis con SIG; en segundo lugar, aportando a estos análisis todos los registros realizados en el campo, lo que nos permitió brindar información adicional a dichos análisis, como así también ajustar los estudios observando coincidencias o falencias de las técnicas numéricas y binarias realizadas por la computadora, sin olvidar además la resolución del MDT que estamos utilizando.

Para el Valle de Balcosna, los análisis de visibilidad buscan responder a preguntas como: ¿existían pautas visuales distintas acorde a las diversas estructuras arqueológicas?, ¿se puede relacionar complejidad arquitectónica con mayor visibilidad?, ¿había necesidad de una intervisibilidad entre los asentamientos en el valle?, ¿el paisaje visual estaba culturalmente pautado?, es decir, ¿existiría en el Valle de Balcosna una estrategia consciente de creación de un paisaje visual que pueda explicar una parte de la variabilidad de la distribución espacial de estos sitios?

\section{Trabajos de campo}

Quisiéramos explicitar aquí cómo se llevaron a cabo los trabajos de prospección y relevamiento de los sitios arqueológicos que luego utilizamos para llevar adelante los análisis de cuencas visuales teóricas.

Los primeros relevamientos comenzaron en el año 2005. En dicha oportunidad el área de prospección fue acotada, por lo que para esta oportunidad la ampliamos con un total aproximado de $46 \mathrm{~km}^{2}$.

En esta ocasión, al realizar las prospecciones se adoptó el

\footnotetext{
${ }^{3}$ Actualmente existen diferentes formas de analizar la visibilidad de un grupo de asentamientos. Los primeros análisis se hacían de forma manual, yendo al campo, obteniendo panorámicas a través de fotos y realizando croquis de orientación; con el tiempo este trabajo fue sustituido por los SIG sin la necesidad de salir al campo, permitiendo la realización de una importante cantidad de análisis que antes era muy difícil o imposible de realizar. La sustitución del trabajo de campo en favor de los análisis computacionales mediante el estudio de mapas en formato raster resultó ser un arma de doble filo, ya que si bien permite obtener una abundante cantidad de información se supone que no hace falta corroborarla en el campo, en este trabajo, en cambio, tomamos en cuenta esto y cada sitio contó con fotos panorámicas tomadas en el campo, algo que sirvió como complemento para los estudios con SIG.
}

criterio de caminar por el paisaje del valle siguiendo los senderos y vías de tránsito actuales. Esta metodología, que llamamos "sistema de prospección por sendas"4, nos ha permitido acercarnos al paisaje de una manera diferente a la acostumbrada por aquellos arqueólogos que simplemente realizan prospecciones lineales (transectas): si bien no se recorre "sistemáticamente" la totalidad del área, una persona puede moverse "naturalmente" a través de ella. Las sendas nos brindan un modo de conocer el paisaje de manera diferente, al permitirnos comprender que algunos lugares que a priori parecían alejados por la distancia y la topografía, son factibles de conectarse mediante sendas que se acomodan a las sinuosidades propias del terreno. Aunque no sabemos si fueron estas sendas (actuales) las que conectaron los sitios y el paisaje en el pasado, la idea general de esta metodología no es explicar esa asociación empírica y directa, sino que nos permita demostrar la transitabilidad y fluidez de los paisajes que recorremos.

En relación a las estructuras, nos basamos en las categorías de Assandri y Laguens (1999) usadas para el vecino Valle de Ambato, quienes las clasificaron de acuerdo a los metros cuadrados construidos. A partir de ello, para el caso del Valle de Balcosna definimos cuatro categorías:

1- Unidades pequeñas: estructuras que varían entre unos pocos metros y $150 \mathrm{~m}^{2}$. Se caracterizan generalmente por ser unidades simples y en algunos casos por tener divisiones internas y/o uno o más recintos adosados.

2- Unidades medianas: estructuras superiores a los $150 \mathrm{~m}^{2}$ que llegan hasta $300 \mathrm{~m}^{2}$. Se distinguen de las anteriores fundamentalmente por el tamaño. También se observan con mayor frecuencia varios recintos adosados y algunos no adosados. En algunos casos éstas estructuras se encuentran cerca de las terrazas de cultivo.

3- Unidades grandes: su tamaño oscila entre $300 \mathrm{~m}^{2}$ y 600 $\mathrm{m}^{2}$. Consisten en una gran diversidad de estructuras, por lo general complejas, con recintos que presentan varias divisiones internas y sectores. Muchas de ellas están asociadas a terrazas de cultivo y morteros.

4- Unidades muy grandes: son aquellas que superan los $600 \mathrm{~m}^{2}$. Se caracterizan por tener recintos grandes y amplios, además de recintos adosados y no adosados alrededor. Generalmente se vinculan con unidades de menor tamaño y terrazas de cultivo.

\section{Como hemos señalado, el "Sistema de Prospección por}

\footnotetext{
${ }^{4}$ La metodología denominada "sistema de prospección por sendas" (SPS) surgió de la necesidad de estudiar los paisajes arqueológicos del Valle de Balcosna siguiendo las sendas que lo surcan, iniciativa que nos ha permitido acercarnos al paisaje de una manera realmente novedosa. Aunque para este trabajo se encuentra apenas esbozada tiene un desarrollo teórico y metodológico mucho más profundo en la Tesis Doctoral de unos de los autores (ver Villafañez 2013).
} 
Sendas" (SPS) pretende ser un método que nos brinde información más allá del mero hallazgo de los vestigios arqueológicos, procurando ofrecer datos significativos acerca del paisaje en general.

Adentrándonos en los trabajos de campo, estos se llevaron a cabo en el transcurso de dos inviernos (debido a la menor vegetación), entre los meses de julio y septiembre. Estas prospecciones fueron realizadas en su mayoría en períodos cortos, muchas veces de tan solo un día o un fin de semana, salvo contadas ocasiones en las que dispusimos de una semana completa. En total, efectuamos más de 50 salidas de prospección y caminamos más de $450 \mathrm{~km}$ por todo el valle.

El SPS toma a las sendas como una guía por donde poder transitar. Sin embargo éste no es un sistema de prospección cerrado y rígido que determina un camino fijo. Si bien el método consiste en desplazarse por las sendas, una de las premisas es que una persona no está atada a ellas y puede salirse cuando crea conveniente recorrer zonas adyacentes. Esto nos permitió encontrar gran cantidad de sitios arqueológicos, más que nada estructuras agrícolas en pendientes o laderas de quebradas, morteros en afloramientos rocosos y arroyos con grandes sistemas para almacenar agua, además del registro detallado de algunas estructuras con paredes ubicadas a varios metros de donde pasaba la senda por la que transitábamos. En síntesis, las sendas fueron la guía fundamental que nos hizo posible interpretar una posible lógica del paisaje en el valle.

Antes de referirnos al emplazamiento de las estructuras debemos mencionar un tema de suma importancia y que se relaciona con el fondo de valle: el avance del cultivo actual en este sector nos ha impuesto una limitación importante en la investigación, pues ha impedido observar con claridad la disposición de las estructuras en el espacio salvo algunas excepciones. Al prospectar en los campos de cultivo hemos relevado y recolectado una importante cantidad de material cerámico, indicio principal para indicar que esta parte del valle habría estado ampliamente habitada.

Fuera de dichas áreas de cultivo actual, las estructuras se disponen a lo largo de las quebradas laterales que permiten el acceso a la línea de cumbres, desde los 1200 msnm hasta punta de cumbre (hasta 1850 msnm). No existen niveles predilectos para las diferentes construcciones, puesto que encontramos estructuras muy complejas y también de gran tamaño en niveles de pastizales de altura o recintos pequeños en niveles inferiores y viceversa.

Es evidente que uno de los criterios fundamentales para la elección de un lugar de emplazamiento fue la cercanía a algún curso de agua (entre $10 \mathrm{~m}$ y $120 \mathrm{~m}$ ), ya que el total de las estructuras se vinculan al río o a un arroyo

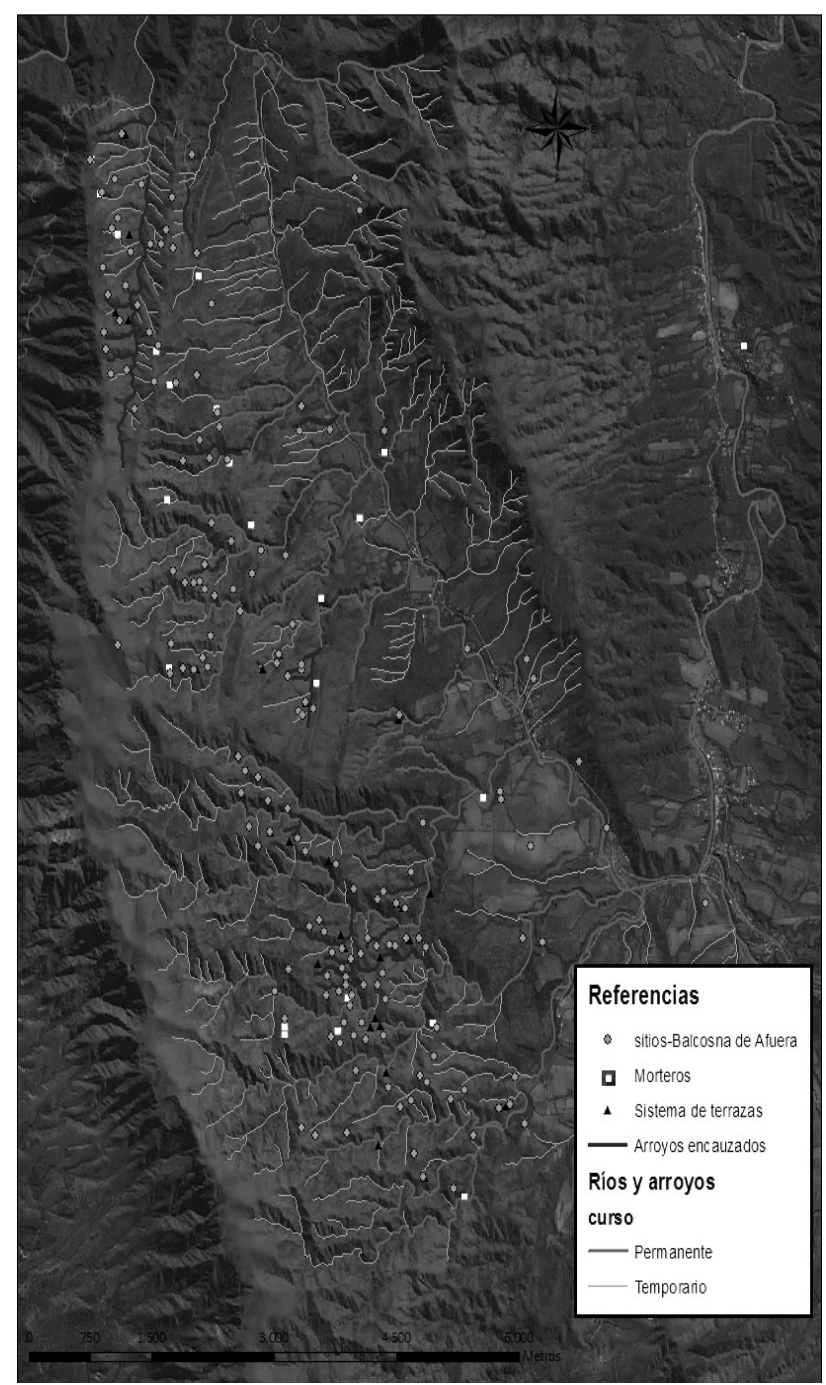

Figura 2: Plano que muestra toda la evidencia arqueológicarelevada junto a los cauces de agua más importantes.

Figure 2: Map showing all the archaeological evidence surveyed along the most important watercourses.

\section{permanente.}

Las prospecciones llevadas a cabo a lo largo del Valle de Balcosna dieron como resultado un total de 164 sitios arqueológicos (ver figura 2) de diferentes tipos, que incluyen posibles unidades de vivienda, corrales, almacenamiento y dispersiones de material cerámico ${ }^{5}$. Además, se hallaron 28 arroyos encauzados, 29 morteros en roquedales naturales y un total de 22 sectores con terrazas de cultivo.

\footnotetext{
${ }^{5}$ Aunque las categorías las agregamos de manera potencial, tenemos evidencia firme obtenida a través de algunas excavaciones realizadas sobre la posible funcionalidad de algunas estructuras. Los trabajos llevados a cabo en el sitio 049, una estructura cuadrangular con dos recintos internos (Ver Villafañez 2012) y las excavaciones en el ya mencionado sitio 111, dan cuenta de un posible corral y de una estructura habitacional. Ademas el análisis bibliográfico de investigaciones cercanas (Ver Figueroa 2007, 2008, 2010), nos han permitido afianzar estas proposiciones sobre las tareas que se habrían llevado a cabo en estos sitios.
} 


\section{Análisis de cuencas visuales teóricas}

Como ya expusimos, la variable de visibilidad consiste, pues, en la cuenca visual potencialmente accesible desde un punto " $x$ " en el paisaje (en nuestro caso los sitios arqueológicos), la cual se registra a través de la distancia máxima visible sin interferencia. Para calcularla, tomamos como máximo un rango de $3 \mathrm{~km}$ de diámetro, que abarca $28,2 \mathrm{~km}^{2}$, lo que nos permitió realizar análisis cuantitativos y así determinar los grados de visibilidad e inter-visibilidad entre sitios. Con ello buscamos saber si existieron diferencias en cuanto a los grados de visibilidad e inter-visibilidad, teniendo en cuenta los tamaños y las posibles funciones de los sitios, evaluando la existencia de indicios de alguna clase de jerarquización o control de determinados puntos en el espacio.

Lo primero que destaca para el Valle de Balcosna son los grandes rangos de visibilidad entre los sitios. Por ejemplo si una persona se encontrara en la estructura que mayor visibilidad posee, tendría un campo visual del 37,31\%, pudiendo ver 26 veces más que si estuviera en el sitio de menor visual, con apenas el 1,44\%. Estas disparidades están presentes a lo largo de todo el valle.

Además debe agregarse que aunque existen muchas diferencias entre los sitios en cuanto a su mayor o menor visibilidad, no se encuentran estructuras con grandes cuencas visuales, ya que son pocas las que apenas superan el $30 \%$ de los $3 \mathrm{~km}$ del diámetro establecido como punto máximo de visión. Aunque es un valle muy encajonado con un acotado fondo de valle y gran cantidad de quebradas y lomas, podría decirse que hubo una elección premeditada por parte de las personas que habitaron el lugar para asentarse en puntos específicos del paisaje, tales como los relieves positivos de lomas y quebradas que tienden a mirar al Norte y al Este. De esta manera, la topografía del valle incidió en el carácter acotado de las cuencas visuales, pero fue la elección de dichos lugares de asentamiento por parte de los habitantes del valle lo que produjo, como expondremos en los análisis a continuación, que existieran condiciones de visibilidad algo acotadas pero de inter-visibilidad muy grandes.

A partir de los análisis realizados con el SIG, hemos diferenciado arbitrariamente cuatro intervalos en relación al porcentaje de $\mathrm{km}^{2}$ que abarcan las cuencas visuales teóricas: 1) más de $30 \%$, 2) 20 a $30 \%$, 3) 10 a $19 \%$ y 4) 1 a $9 \%$.

\section{Rango visual mayor al $30 \%$}

Existe un total de 11 sitios que superan el rango visual de $30 \%$ (figura 3.1), todos ellos ubicados en la parte Sur del valle, que es la más amplia. Son 6 los sitios que están en el fondo de valle y el resto se ubica en la parte inicial de la cumbre, a no más de 1500 msnm.

El sitio con mayor cuenca visual es el 51 y consiste en una pequeña estructura rectangular, situada sobre una estrecha loma en la Cumbre del Contador. Esta disposición hizo posible que desde allí pueda observarse con gran nitidez el fondo de valle y gran parte de la Cumbre de Balcosna, al Oeste.

Otro sitio de importancia en relación a la visual es el número 5 , el cual se encuentra en una zona realmente estratégica al estar ubicado en una loma de ingreso al valle, donde confluyen los ríos de Balcosna y de Potrerillos. Se trata del lugar en que comienza la Cumbre del Contador, donde el valle se desdobla y de cada lado de la misma se disponen las localidades de la Villa de Balcosna y Balcosna de Afuera. Aunque los datos generados por el SIG muestran que desde el punto donde se generó el raster se obtiene más visual hacia el Este, pudimos corroborar en el campo que desde otros puntos de la loma (que posiblemente fue parte del mencionado sitio) existe un control panorámico total del ingreso hacia el Valle de Balcosna.

En cuanto a la parte media de la Cumbre de Balcosna, de no más de 1500 msnm, existen algunos sitios que tienen mayor visibilidad, los cuales están muy cerca uno de otro. Se trata de estructuras poco complejas, dos de ellas posiblemente pequeños corrales y una que hemos interpretado como una estructura de habitación, de gran tamaño. Desde esa posición, una persona logra ver muchos sitios cercanos, además de casi la totalidad del fondo de valle.

Entre los sitios más complejos con una buena visibilidad, se encuentra el sitio 116, que cuenta con más de 6 sectores y 20 recintos adosados y no adosados, ocupando más de $80 \mathrm{~m}$ a lo largo de una loma en dirección NorteSur. Tiene una excelente vista del fondo de valle y de la parte sur de la cumbre, logrando ver más elevada de la misma (figura 3.2).

Debido a la referida cercanía de estos sitios entre sí, realizando la sumatoria de visibilidades de los 11 sitios considerados, pudimos apreciar que tienen mayor visibilidad de la parte centro y sur del fondo de valle, donde prácticamente no existe un punto de vista ciego. En cambio, esa visibilidad va disminuyendo cuando consideramos la parte media y alta de la cumbre, haciéndose muy difuso hacia niveles que superan los $1700 \mathrm{msnm}$. Solo en casos muy puntuales algunos sitios (p.e. 44, 45, 116) son capaces de ver arriba de los 1800 msnm. Es decir que, cuanto más alto en la cumbre se encontraban las personas, menos visual tenían. La necesidad de contar con amplias panorámicas visuales estuvo dada también por la tendencia a asentarse en la parte baja del valle.

En cuanto a la inter-visibilidad, puede decirse que los sitios del fondo de valle son los que menos inter-visibilidad tienen entre sí, ya que no hay tantos y se hallan muy 


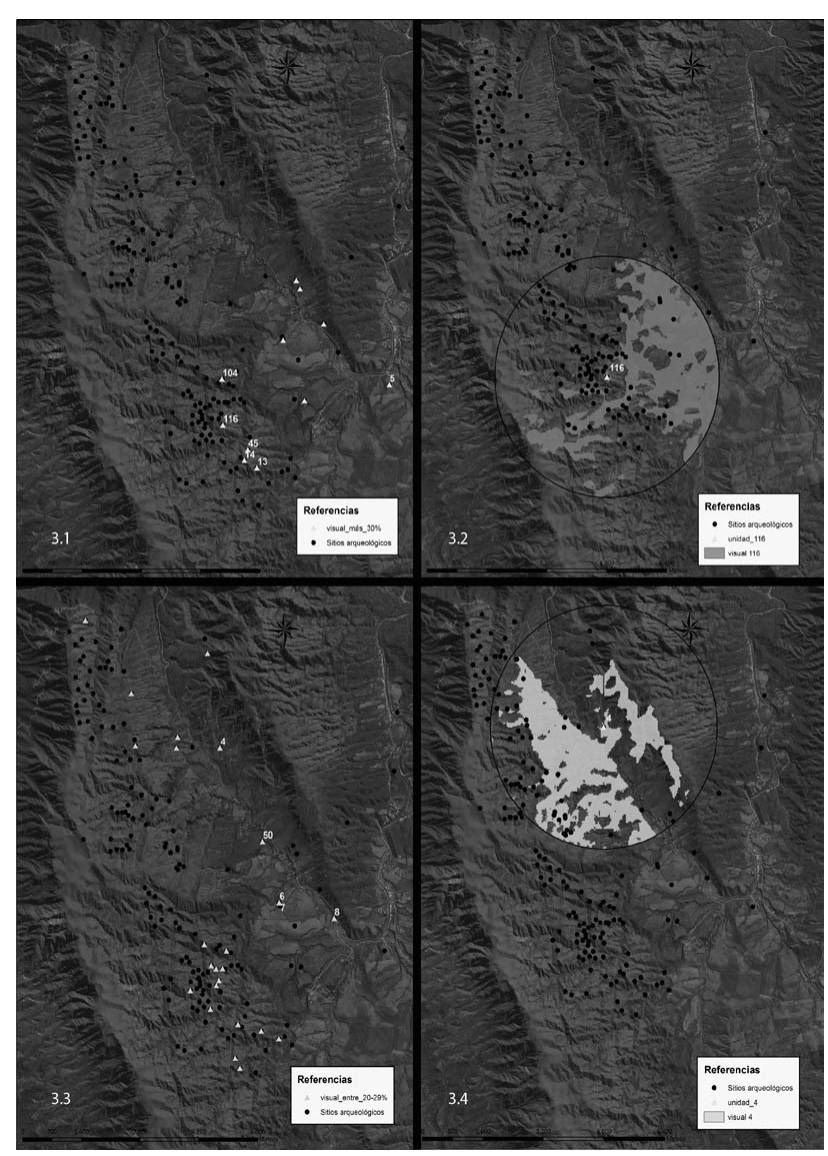

Figura 3: 1: Los triángulos muestran donde se encuentranlos sitios desde donde la visual supera el 30\%. Fig 3.2:Cuenca visual teórica desde el sitio 116, se observa que existe una excelente visibilidad hacia el fondo de valle, con gran inter-visibilidad con los sitios circundantes. Fig: 3.3: Vista general de los sitios con cuencas visuales teóricas entre 20 y 29\%.Fig: 3.4: Cuenca visual teórica desde el sitio 4. Al encontrarse en el fondo de valle tiene un buen control visual hacia la cumbre al oeste.

Figure 3: 1: The triangles show where are the sites from where the visual exceeds 30\%. Fig 3.2: Theoretical visual basin from site 116, it is observed that there is an excellent visibility towards the bottom of the valley, with great inter-visibility with the surrounding sites. Fig: 3.3: Overview of sites with theoretical visual basins between 20 and 29\% .Fig: 3.4: Theoretical visual basin from site 4. Being in the valley bottom has a good visual control towards the summit to the west.

dispersos. Ocurre todo lo contrario en la Cumbre de Balcosna, donde la cercanía entre estructuras hace posible un vínculo visual más cercano, puesto que existe una gran inter-visibilidad, donde las personas en promedio podrían ver y ser vistas por un promedio de 35 sitios.

Rango visual entre 20 y $29 \%$

Los sitios que están en el rango de visibilidad entre 20 y $29 \%$ son 24 y duplican a los anteriores en cantidad (figura 3.3). En relación a su disposición en el espacio, 17 se encuentran en la parte sur del valle; de los 7 restantes, 2 se ubican en la Cumbre del Contador, 1 en el extremo Norte, 2 en la parte baja del fondo de valle y 2 sitios en la parte media de la Cumbre de Balcosna.

El sitio con mayor cuenca visual teórica es el 4, el cual se encuentra al Norte en una loma de la parte baja de la Cumbre del Contador. Desde allí se obtiene una excelente vista panorámica hacia la Cumbre de Balcosna. Además al fondo de valle, al sur, cuenta con una excelente intervisibilidad con 20 sitios arqueológicos (figura 3.4).

Otro sitio relevante en cuanto a la visibilidad en este rango es el 6, ubicado al sur en el fondo de valle, a pocos cientos de metros del sitio 7; el mismo tiene un excelente grado visual hacia la parte norte y este del valle, observando en promedio cerca de 20 estructuras. De los sitios en la zona sur, sólo 3 de ellos aparecen en la parte más baja: el sitio 6 junto al 8 y 50, estos dos últimos con una amplia visual hacia la Cumbre de Balcosna. Los sitios, por lo general, se encuentran en alturas que no superan los 1650 msnm, salvo casos excepcionales como el 132, el 150 o el 158.

Los sitios de este rango visual se disponen de manera más dispersa en el valle y suman un amplio porcentaje de visibilidad respecto al total del área. Observando la sumatoria, puede apreciarse que estos sitios miran la totalidad de la Cumbre del Contador y el fondo de valle, además de la Cumbre de Balcosna; sin embargo la visual sólo alcanza los 1700 msnm, salvo la parte sur, desde donde de algunas zonas pueden ver hasta punta de cumbre.

Rango visual entre 10 y $19 \%$

Los sitios que tienen un rango visual entre 10 y $19 \%$ ocupan niveles altitudinales más elevados, llegando a estar arriba de los 1700 msnm. En total suman 49 y siguen criterios parecidos a los rangos visuales ya analizados. Entre ellos, son 28 los que se ubican en la parte Sur, 2 en el fondo de valle y el resto en la cumbre. Son 13 los que están dispuestos en la parte Centro-Norte y 9 en el extremo Norte, lugar donde tienen una excelente visual hacia el Valle de Escaba, la Cumbre del Aconquija y parte del Valle de Ambato (figura 4.1).

El sitio con mayor visibilidad es el 39. Se trata de una serie de estructuras grandes y complejas ubicadas en la parte norte, lugar privilegiado para observar el comienzo del Valle de Escaba. En este sentido, este sitio tiene un excelente control visual sobre la Senda de la Cumbrecita, paso seguro y tradicional hacia Escaba. Sin embargo, al mismo tiempo, la observación está limitada hacia el Valle de Balcosna (figura 4.2). En cuanto a la inter-visibilidad, son 14 los sitios con los que tiene contacto visual.

Otro sitio de importancia es el 193, dispersión cerámica que se ubica en el estrecho fondo de valle del Norte, a pocos cientos de metros del sitio 4. Tiene un buen control visual sobre la Cumbre del Contador y parte de la Cumbre de Balcosna, pero está imposibilitado de ver hacia el norte y sur, en tanto que tiene inter-visibilidad con 8 sitios.

Algunos sitios que se encuentran al sur en el fondo de valle, son también de importancia en relación al rango 


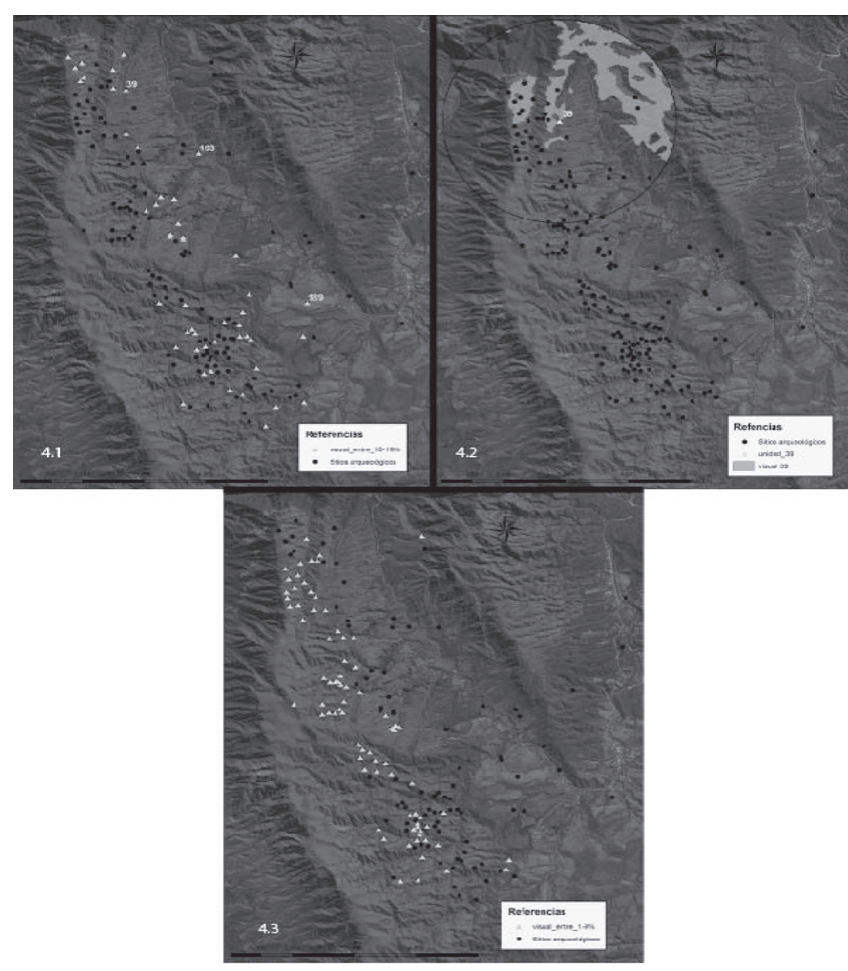

Figura 4: 1: Vista general de los sitios con cuencas visuales teóricas entre 10 y 19\%.Fig 4.2:Cuenca visual teórica del sitio 39. Con un excelente control visual hacia el norte e inter-visibilidad hacia lossitios de las cumbres cercanas. Fig 4.3: Vista general de los sitios con cuencas visuales teóricas entre 10 y $19 \%$

Figure 4: 1: Overview of sites with theoretical visual basins between 10 and 19\% .Fig 4.2: Theoretical visual basin of site 39. With an excellent visual control towards the north and inter-visibility towards the sites of nearby peaks. Fig 4.3: General view of sites with theoretical visual basins between 10 and $19 \%$.

visual al que estamos refiriendo. Aunque se ubican en la parte más baja y abierta del valle, debido a su emplazamiento particular en la zona, está imposibilitado de ver hacia el norte y el sur, pero en cambio tiene una excelente visual hacia ambas cumbres al este y oeste, lo cual le permite tener una buena inter-visibilidad con más de 30 sitios.

\section{Rango visual entre 1 y $9 \%$}

Los sitios que tienen los porcentajes de visibilidad más bajos son 79 en total. En comparación con los demás, estos se disponen de una manera homogénea a lo largo del valle. No existe ninguno de estos sitios en el fondo de valle, repartiéndose 23 en el sur y norte y 33 en el centro (figura 4.3).

La mayor cantidad de sitios que componen este rango visual son aquellos que están emplazados en la zona central. Esto podría explicarse por el carácter encajonado del valle en esta parte, lo que impide amplias panorámicas visuales.

A través de estos análisis, volvemos a reforzar el principio de que a mayor altura sobre el nivel del mar los sitios tienen menor cuenca visual teórica. Por lo general, todos los sitios tienen un rango visual disperso, sin lograr una continuidad en las visuales. Siguiendo el registro de los raster generados por el SIG, podemos decir que estos sitios ven "por parches", ya que por lo general están ubicados en hondonadas o zonas con importantes impedimentos visuales, como lo son las grandes quebradas o laderas.

Realizando un resumen general de todas las cuencas visuales teóricas, algunas cuestiones son interesantes de destacar. Por una parte, puede decirse que la visibilidad va disminuyendo de sur a norte. La zona sur del valle es la más accesible visualmente debido a que en promedio tiene un ancho de 2000 m; en el Norte en cambio, al ir encajonándose el valle al punto de llegar a tener menos de 100 m, las visuales disminuyen drásticamente.

Por otra parte, la visual también disminuye desde el fondo de valle hasta punta de cumbre. Al contrario de lo que puede pensarse, en líneas generales cuanto a más elevado respecto el nivel del mar se encuentra un sitio menos puede ver, lo cual se debe en parte al carácter quebradizo de la zona cumbral, que en muchos casos forma barreras naturales que cortan las visuales.

En cuanto a la inter-visibilidad, en promedio, los sitios que tienen una visibilidad de media a baja son los más inter-visibles con el resto. En este punto tenemos que considerar, primero, que el fondo de valle es el lugar con más probabilidad de ver, pero al tener pocas prospecciones debido a la gran alteración antrópica existe un sesgo importante que debemos remarcar; segundo, que la cercanía de estructuras presentes en la cumbre de Balcosna, ha hecho posible un vínculo mucho más estrecho en cuanto a la inter-visibilidad.

\section{Algunas consideraciones sobre la relación entre la visibilidad y el tamaño de los sitios.}

Creemos conveniente preguntarnos en este punto si podemos ver alguna lógica de visibilidad según el tamaño, ¿las personas en los sitios de mayor tamaño tenía mayor visibilidad?; o también cómo se relaciona la visibilidad con las posibles funciones, ¿existió alguna lógica de visibilidad relacionada a los sitios de hábitat?

Si tenemos en cuenta los rangos de visibilidad con respecto al tamaño, de los sitios con visuales mayores al $30 \%$, que suman 11, 5 son pequeños, 1 mediano, 2 grandes y 3 muy grandes. A mayor visibilidad, entonces, no necesariamente existe mayor tamaño y complejidad en los sitios.

Considerando la visibilidad en relación con las posibles funciones de dichos sitios, tenemos que 6 son de hábitat, 1 se trataría de un corral y 4 son dispersiones de material cerámico a las que no se les pudo asignar una posible función.

En el rango visual entre 20 a $29 \%$, de los 24 sitios que lo componen 12 son pequeños, 7 medianos, 3 grandes y 2 


\begin{tabular}{lll}
\hline Sitio & Tipo de sitio & Posible función \\
\hline 4 & Pequeño & Habitacional \\
5 & Muy Grande & Dispersión cerámica \\
6 & Pequeño & Habitacional \\
7 & Pequeño & Habitacional \\
8 & Pequeño & Dispersión cerámica \\
39 & Grande & Habitacional \\
44 & Muy Grande & Habitacional \\
45 & Pequeño & Habitacional \\
50 & Pequeño & Corral \\
51 & Pequeño & Habitacional \\
91 & Mediano & Corral \\
100 & Muy Grande & Corral \\
104 & Mediano & Habitacional \\
116 & Grande & Habitacional \\
132 & Pequeño & Habitacional \\
150 & Grande & Corral \\
158 & Pequeño & Corral \\
193 & Muy Grande & Habitacional \\
\hline
\end{tabular}

Tabla 1: Se muestran todos los sitios arqueológicos mencionados en el trabajo, destacándose el tipo de sitio según el tamaño y la posible función de los mismos.

Table 1: All the archaeological sites mentioned in the work are shown, highlighting the type of site according to the size and the possible function of the same. muy grandes. Esto muestra una continuidad respecto al tamaño de los sitios del rango anterior, en el sentido de que no necesariamente a mayor tamaño se corresponden mayores visuales.

En cuanto al rango de 10 a $19 \%$, de los 49 sitios se observan los siguientes tamaños: 21 pequeños, 10 medianos, 12 grandes y 6 muy grandes. Considerando esta distribución, podría decirse que a medida que la visibilidad se hace más acotada, la cantidad de sitios por tamaño disminuye proporcionalmente.

Por último, en el rango de 1 a $9 \%$ tenemos que del total de 79 sitios, 40 son pequeños, 19 medianos, 14 grandes y 6 muy grandes.

Si tomamos en cuenta los 15 sitios muy grandes, solo 3 sobrepasan el $30 \%$ de cuenca visual teórica, 1 llega al 22 $\%$ y el resto tiene menos de $20 \%$. Un ejemplo de ello es el sitio 100, que es uno de los más grandes y complejos del valle. Ubicado a 1800 msnm y ocupando la totalidad de una loma, es el antepenúltimo sitio con menor visibilidad de todo el valle: con apenas una cuenca visual de 1,5 \% observa sólo las quebradas vecinas del norte y tiene un escueto control visual de la Senda de los Varela, al sur.

\section{Discusión y consideraciones finales}

Antes de comenzar a desarrollar este acápite, quisiéramos hacer referencia a la cronología, puesto que al llevar a cabo un análisis de índole espacial y caracterizar una serie de estructuras a lo largo de todo un valle, se esperaría que exista algún grado de contemporaneidad. Debemos decir que tenemos una sola datación de carbono 14 en uno de los sitios estudiados por medio del fechado por AMS de una muestra de madera carbonizada, obtenida de una estructura de combustión en uno de los recintos excavados (E111), ubicándolo temporalmente en 1360 +/- 3 AP ${ }^{6}$ (Ver Villafañez 2012). Aunque con un solo dato

\footnotetext{
${ }^{6}$ La muestra enviada al Laboratorio de "NSF Arizona AMS Facility.
}

cronológico absoluto, existen otros indicadores relativos que nos aportan datos relevantes, uno de ellos es el material cerámico recolectado, en la mayoría de los sitios relevados se ha registrado cerámica Aguada Ambato, que se enmarca dentro de la datación obtenida, otro dato de importancia es el análisis de la arquitectura, ya que todos los sitios presentan una morfología parecida, muchas estructuras se repiten en el espacio ya sea en su forma, material y ubicación en el paisaje, esto nos lleva a pensar que en algún momento, muchos de las estructuras fueron contemporáneas.

En relación a la visibilidad, pudimos interpretar que a mayor altura existe menor rango visual, por lo que el fondo de valle es privilegiado en ese aspecto, principalmente la parte sur, debido a que el norte es mucho más angosto y elevado.

Para categorizar la visual de los sitios decidimos realizar una delimitación arbitraria en cuanto al porcentaje de las cuencas visuales teóricas, definiendo 4 intervalos: 1) más de $30 \%$, 2) 20 a $30 \%$, 3) 10 a $19 \%$, 4) 1 a $9 \%$. A continuación se sintetizan los resultados de estos análisis.

Todos los sitios con mayor rango visual se registraron al sur, donde el fondo de valle es más ancho y presenta mayor posibilidad de panorámicas visuales, siendo los más relevantes en este aspecto aquellos que se encuentran en la parte baja de la Cumbre del Contador. Aún en el sur, los sitios con alto porcentaje de cuenca visual teórica en ningún caso superan el $37 \%$. Estos se encuentran en las primeras lomas de la cumbre, destacándose los sitios grandes y medianos, ambos bastantes complejos como son los sitios 104 y 116 . Es de destacar también el sitio 5 ubicado en el extremo sur, el cual habría tenido un control visual desde el inicio del valle.

Hay que realizar algunas advertencias al referirnos a las implicancias que pudo tener el control visual en el fondo

The University of Arizona EE.UU", ha proporcionado una ubicación temporal del recinto en el siglo VII DC, cuya datación por AMS dio como resultado una edad 1360 +/- 3 AP (NSF-1644). 
de valle: por un lado, debe aclararse que no hubo la misma intensidad de prospecciones en esta zona a causa del cultivo actual, lo que ha propiciado que si bien en dicha zona se encuentren los sitios con más visibilidad no sucede lo mismo respecto a la inter-visibilidad, ya que se han registrado muy pocos, al contrario que en la cumbre; por otro lado, en relación con la masa arbórea, es de esperar que en épocas pasadas la vegetación en la cumbre haya sido la misma que la actual, por lo que los pastizales no habrían significado un inconveniente para la visibilidad, algo que no podemos predecir para la parte de fondo de valle, pues posiblemente en el pasado la vegetación (que hoy es escasa debido a la agricultura extensiva) haya sido un elemento que funcionó como limitante; es por eso que este estudio versa sobre las cuencas visuales teóricas.

Siguiendo con los análisis, los sitios con visuales entre 20 y $29 \%$ duplican a los anteriores en número, pero la lógica sigue siendo la misma: 17 en el sur, 2 en la parte central del valle, otros 2 sobre la cumbre del Contador y 1 en el extremo norte, zona de amplias visuales hacia los Altos de Singuil en Ambato y el Valle de Escaba. El más relevante de este rango es el sitio 4, ubicado al pie de la Cumbre del Contador, siendo el lugar donde se encontró un suplicante.

En cuanto a los dos últimos rangos visuales que analizamos, o sea de 10 a $19 \%$ y de 1 a $9 \%$, puede decirse que a más metros sobre el nivel del mar, menor es la cuenca visual en los sitios. Aquellos sitios que cuentan con menor visibilidad se concentran en la parte central del valle, lugar de visual más restringida si se lo compara con el extremo Norte, que tiene amplias visuales hacia Escaba, la Cumbre del Aconquija y los Altos de Singuil en Ambato.

¿Qué rescatamos de este análisis en el Valle de Balcosna? ¿Qué nos dicen los patrones visuales que hemos descubierto?

En Balcosna nos encontramos con un valle "salpicado" de viviendas, corrales para la cría de animales, lugares para almacenamiento de alimentos y roquedales plagados de morteros, todos dispuestos siempre cerca de algunas de las decenas de arroyos que descienden de la cumbre al río y ubicados desde el fondo de valle hasta punta de cumbre, mostrando así una continuidad en el uso del espacio. Con seguridad, sea donde fuera que una persona estuviera, y debido a la cercanía entre las estructuras, ésta siempre podía ver o ser vista por otro grupo de individuos.

Correlacionando estos datos con el tamaño vemos que no necesariamente a mayor tamaño se da un mayor control visual. Si bien podemos seguir la linealidad de los datos en cuanto a que los sitios del fondo de valle, donde se ubican las estructuras más grandes, son aquellos que más visibilidad tienen, debemos aclarar primero que aún no conocemos cómo fue la vegetación en el pasado; creemos que debió existir la suficiente para impedir la posibilidad de panorámicas visuales como en la actualidad, por lo que los datos deberían ser interpretados con cuidado. Además, debe recordarse que existen sitios ubicados en las partes más elevadas de la cumbre, realmente significativos en cuanto a su tamaño e importancia, pero que no tienen gran control visual, como son el 100, el 91 o el 44. Aunque existen casos de poco control visual, la inter-visibilidad nunca se ve afectada por barreras naturales. En promedio, desde cada sitio una persona podría observar a otros 20 que lo rodean.

Para nosotros fue importante escapar de las concepciones actuales en cuanto al paisaje puramente occidental y moderno, puesto que creemos que en el pasado premoderno y no occidental no hay sistema de vigilancia de tipo panóptico como los descriptos por Foucault (1975).

Así, lo significativo de este análisis no puede resumirse al área en $\mathrm{km}^{2}$ de visibilidad desde los sitios, sino a quiénes y a qué se podía ver desde ellos. Es decir que, si bien no existen grandes panorámicas visuales (recordemos que desde el sitio que más panorámica visual se tiene no supera el $37 \%$ ), se destaca la inter-visibilidad que existe, puesto que del total de 164 sitios analizados, salvo 5 de ellos, el resto es capaz de ver y ser visto. En total nos encontramos con una inter-visibilidad entre sitios del 99 $\%$, o sea que prácticamente cualquier persona ubicada en cualquiera de los sitios tuvo una gran visibilidad. En promedio, un sitio es inter-visible con por lo menos 12 que lo rodean. Dicho de otro modo, los sitios están dispuestos en el paisaje no necesariamente para ver mucho, sino simplemente para que las personas que los habitaron a lo largo del valle se viesen entre sí, estando en permanente contacto, ya sea con la mirada.

San Fernando del Valle de Catamarca, 5 de agosto de 2016

\section{Bibliografía}

Assandri, S. y A. Laguens 1999. Asentamientos aldeanos Aguada en el Valle de Ambato. Actas del XIII Congreso Nacional de Arqueología Argentina, Córdoba. En prensa.

Albeck, M. 1994. La quebrada de Humahuaca en el intercambio prehispánico. En: Taller de Costa a Selva. M. E. Albeck (Ed.), pp. 117-137. Instituto Interdisciplinario Tilcara, Universidad de Buenos Aires, Tilcara.

Barrionuevo, A. y J. Schobinger 1992. Observaciones sobre algunos lugares arqueológicos del valle de Catamarca y alrededores. En: Shincal 2. pp. 95-99.

Cabrera, A. 1976. Regiones Fitogeográficas Argentinas. En: Enciclopedia argentina de agricultura y jardinería. Tomo II. Fascículo 1, pp. 1-85ACME, Buenos Aires. 
Cattania, S. y M. Varela 2010. Algunas consideraciones geográficas del departamento Paclín. En Por las sendas de Paclín. En: Estudios Sociales de un Departamento Catamarqueño, editado por Emilio Villafañez y Marcelo Gershani Oviedo, pp. 11-22. Sarquís. Catamarca. En Prensa.

Conolly J., M. Lake 2009. Sistemas de información geográfica aplicados a la arqueología. Ediciones Bellaterra. España.

Clarke D. 1977. Spatial archaeology. Academic Press.

Criado Boado F. 1993. Límites y posibilidades de la arqueología del paisaje. En: Spal 2, pp. 9-55.

Criado Boado F. 1995. Construcción social del espacio y reconstrucción arqueológica del paisaje. En: La perspectiva espacial en arqueología, editado por Barros y Nastry, pp. 75-116. Centro Editorial América Latina. Buenos Aires.

Fábrega Álvarez, P. 2005. Tiempo para el espacio. Poblamiento y territorio en la Edad del Hierro en la comarca de Ortegal (A Coruña, Galicia). En: Complutum, Vol. 16: 125-148.

Figuerero Torres M. y A. Izeta 2013. El uso de sistemas de información geográfico (SIG) en la arqueología sudamericada. Archaeopress, Oxford.

Figueroa G. 2007. Sistema de producción agrícola en la vertiente occidental del valle de Ambato, Catamarca, siglos VII a XI d.C. Informe inédito.

Figueroa G. 2008. Los sistemas agrícolas del Valle de Ambato, Catamarca, siglos VI a XI d.C. En: Intersecciones en Antropología N 9: 313-317.

Figueroa G. 2010. Organización de la producción agrícola en contextos sociales no igualitarios: el caso del valle de Ambato, Catamarca, entre los siglos VII y XI D.C. Tesis de Doctorado en Historia inédita. FFyH, Universidad Nacional de Córdoba.

Foucault 1975. Vigilar y castigar. Nacimiento de la prisión. Siglo XXI.

Garay De Fumagalli, M. 1994. Relaciones de complementariedad en el período de desarrollos regionales entre el ámbito de Valles orientales y en la Quebrada de Humahuaca en el sector centromeridional (Quebrada de Humahuaca, Prov. de Jujuy, Argentina). En: Taller de Costa a Selva. Instituto Interdisciplinario Tilcara, Facultad de Filosofía y Letras. UBA.

García Sanjuán, L. 2005. Introducción al reconocimiento y análisis arqueológico del territorio. Ed. Ariel.

García Sanjuán, L. 2006. Análisis de pautas de visibilidad en la distribución de monumentos megalíticos de Sierra Morena Occidental. En: La aplicación de los SIG en la Arqueología del Paisaje. Alicante, editado por Grau Mira, pp. 122-157. España.

González, A. 1977. Arte precolombino de la Argentina. Introducción a su historia cultural. Buenos Aires, Filmediciones Valero.

González, A. 1961-64 La Cultura de La Aguada del N. O. Argentino. Revista del Instituto de Antropología, $\mathrm{N}^{\circ} 2$ et 3 Universidad Nacional de Córdoba.

Grau Mira, I. 2006. Transformaciones culturales y modelos espaciales. Aproximación SIG a los Paisajes de la romanización. En: La aplicación de los SIG en la Arqueología del Paisaje, editado por Grau Mira, pp. 211-226. Alicante.

Grau Mira, I. 2011. Movimiento, circulación y caminos en el paisaje digital. La aplicación de los sig en el estudio arqueológico de los desplazamientos humanos. En: Actas del V Simposio Internacional de Arqueología de Mérida, pp. 369-382.

Hamilakis 2015. Arqueología y los sentidos. Experiencia memoria y afecto. JAS arqueología.

Hiernaux, D. y A. Lindón, 2006. Introducción. La geografía humana: un camino a recorrer. En: Tratado de Geografía Humana, editado por Daniel Hiernaux y Alicia Lindón, pp. 7-22. Anthopos. Mexico.

Hodder, I. y C. Orton 1990. Análisis espacial es arqueología. Crítica, Barcelona.

Ingold, T. 1993. The Temporality of the Landscape. World Archaeology 25: 152-174.

Ingold, T. 2000. The Perception of the Environment. Routledge. London and New York.

Kriscautzky, N. 1995. Avances en la arqueología del formativo inferior en el Valle de Catamarca. En: Revista de Ciencia y Técnica, Vol. 2. pp. 65-82. Ed. UNCA. Catamarca. Argentina.

Kriscautzky, N. 2006. El Arte al Pie de los Andes Antes de la Conquista Arqueología de Catamarca y el Noroeste argentino. En: Tesoros precolombinos del Noroeste argentino, editado por Matteo Goretti, pp. 47-61.

Lanzelotti 2013.La evaluación multicriterio en el espacio regional y dimensión histórico-arqueológica. En: Teoría y métodos de la geografía cuantitativa. Libro 1: Por una 
geografía de lo real. Editado por Gustavo Buzai, Graciela Cacace, Luis Humacata, Sonia Lanzelotti. Pp. 93-104. MCA Libros.

Llobera, M. 2003. Extending GIS-based visual analysis: the concept of visualscape. En: International Journal of Geographical Information Science, 17: 25-48.

Morlans, M. 1995. Regiones naturales de Catamarca Provincias geográficas y provincias fitogeográficas. En: Revista de ciencia y técnica. pp. 1-36. UNCA. Catamarca. Argentina.

Nogué I Font, J. 1985. Geografía humanista y paisaje. En: Anales de geografía de la Universidad Complutense $n^{\circ}$ 5: 93-107.

Ortega Valcárcel, J. 2004. Los horizontes de la geografía: teoría de la geografía. Ed. Ariel.

Parcero-Oubiña C.; Fábrega-Álvarez, P.; Güimil Fariña A.; Fonte J.; Y J. Valdez. 2009. Castros, caminos, rutas y ocupación del espacio. Modelización y análisis de las formas de movilidad asociadas a los asentamientos de la Edad del Hierro a través de herramientas SIG. En: Arte rupestre, paleoambiente y paisaje. Miradas interdisciplinares sobre Campo Lameiro. Col. TAPA, editado por Felipe Criado Boado, Antonio Martínez Cortizas, Santiago de Compostela: CSIC.

Raffino, R. 1997. Los suplicantes del Museo de La Plata. Corpus Antiquitactum Americanensium. Argentina, I. Union Académique Internationale, Academia Nacional de la Historia. Buenos Aires.

Sanguin, A. 1981. lagéographiehumanieuol'approcheph énomenologique des lieux, des paysages et des espaces. Annales de géographie, $n^{\circ}$ 501: 586-584. Francia.

Tilley C. 1994. A Phenomenology of Landscape: Places,
Paths, and Monuments. Berg. Oxford.

Ventura, B. 1994. Un verde horizonte de sucesos. En Taller de Costa a Selva. Instituto Interdisciplinario Tilcara, Facultad de Filosofía y Letras. pp. 128-144. Universidad de Buenos Aires.

Ventura, B. 2001. Los últimos mil años en la arqueología de las yungas. En Historia Argentina Prehispánica, editado por Berberián y Nielsen, pp. 447-492. Ed. Brujas. Córdoba.

Villafañez, E. 2007. Arqueología Espacial del Valle de Balcosna. Departamento Paclín, Provincia de Catamarca. En http://www.editorial.unca.edu.ar/digitesis.htm. (Acceso 10 de marzo 2012).

Villafañez, E. 2011. Entre la geografía y la arqueología: el espacio como objeto y representación. En Revista de Geografía Norte Grande n50, Vol. 1 pp. 135-150. Arica.

Villafañez, E. 2012. Arqueología, senderos y paisaje en el valle de Balcosna. En: La Zaranda de Ideas. Revista de jóvenes investigadores en arqueología. N 8: 119-136. Buenos Aires.

Villafañez, E. 2013. Espacio y paisaje entre el Cañón de Paclín y el Valle de Ambato, provincia de Catamarca. Tesis doctoral.

Villafañez E., Fonseca E., Acuña G., H. Puentes 2015. Moviéndose con el paisaje: una propuesta metodológica desde el valle Balcosna, Catamarca. En: Relaciones de la Sociedad Argentina de Antropología. $\mathrm{N}^{\circ} \mathrm{XL}$ volumen 2, pag. 133-152.

Wynveldt F., Balesta B. y M. Iucci 2013. El paisaje tardío del Valle de Hualfin: Una reconstrucción arqueologica desde los poblados protegidos. En:Comechingonia, revista de arqueología. N¹7, tomo II, pp. 191-216.CEH. 